Paul Biolley (1862-1908), fue un destacado educador suizo, que llegó a Costa Rica en 1886, durante la administración de Bernardo Soto, en la que fungía como Secretario de Instrucción Pública Mauro Fernández. Fue contratado como profesor de la denominada entonces Escuela Normal y Modelo, que se transformó luego en el Liceo de Costa Rica. Desarrolló una muy notable labor docente en el campo de las ciencias naturales, y muchos de sus trabajos sobre Costa Rica gozan aún de prestigio y valor. Les dio un innegable impulso a las ciencias naturales, mediante variadas y constantes investigaciones; entre ellas sobre los moluscos dela Isla del Coco, sobre los invertebrados y otros temas más. También fue un notable traductor de obras científicas, sociales e históricas, varias de ellas relacionadas con Costa Rica.

En 1889 publicó en París, como fruto de su indudable interés por el presente y el futuro de nuestro país, la obra Costa Rica et son avenir [Costa Rica y su porvenir]. Es un estudio sobre el país en general, sus habitantes, sus zonas geográficas, sus rasgos culturales, su industria, comercio y hacienda. El capítulo final, «L'avenir», es de singular agudeza. Ya con tres años de haber vivido en Costa Rica, estuvo en condiciones de detectar aspectos esenciales de la historia nacional, del talante de sus pobladores y de muchos otros aspectos que, leídos desde hoy, parecen haberse escrito en nuestros días. Se trata de uno de los primeros documentos, escritos en lengua francesa, sobre la historia y la cultura costarricenses, de especial valor por dar cuenta de la percepción que de la nación se tenía a finales del siglo XIX.

LETRAS rescata, en esta sección «Documentos», una muestra escrita del legado de un brillante pedagogo y pensador, quien, como 
muchos otros extranjeros, se afincó en nuestro medio y trabajó con lealtad y generosidad en su nueva patria que fue, en el caso de Biolley, la definitiva. La Dirección de esta revista les agradece a los encargados de la Biblioteca de Libros Antiguos y Especiales, de la Universidad Nacional, haber permitido reproducir este documento, que forma parte del acervo bibliográfico de esa valiosa colección. 


\section{Costa Rica et son avenir}

\section{Paul Biolley}

\section{Chapitre vi. L'avenir}

Nous avons cherche, dans les chapitres précédents, à exposer les faits d'une façon consciencieuse et vraie. Il ne nous semble pas inutile maintenant d'en tirer quelques conclusions pratiques. Notre travail a un double but : d'une part, dissiper un certain nombre d'erreurs géographiques ou scientifiques, ainsi que beaucoup d'erreurs de jugement, sur Costa Rica ; d'autre part, attirer l' attention des émigrants européens sur cette république encore à peu prés inconnue. Quoique le tableau que nous avons essayé de tracer du pays et de ses habitants reste forcément incomplet, ne soit même qu' une légère esquisse, nous ne croyons pas devoir insister encore sur ce premier point. Nous avons surtout tenté de donner la note juste, c'est-à-dire voulu montrer Costa Rica tel qu'il est aujourd'hui. Nous avons, pour cela, puisé aux sources les plus sûres ; nous avons contrôlé la plupart des renseignements qu' elles nous fournissaient ; nos appréciations et nos jugements enfin, que nous n'exposons pas, d'ailleurs, comme indiscutables, sont dictés par l'expérience qu'un séjour suffisant dans le pays nous a permis d'acquérir. L'étude de Costa Rica reste encore à faire ; ce n'est que dans plusieurs années que les matériaux aurontété rassemblés en assez grand nombre pour permettre d'écrire un livre complet ; nous ne revendiquons, pour notre part, que le mérite modeste d'avoir, après d'autres, planté un nouveau jalon dans la voie à ouvrir.

La question de l'émigration est trop importante pourque nous ne nous efforçions pas de mettre mieux en lumière quelques-unes des conclusions qui doivent ressortir des faits exposés dans le cours de 
cette étude. « De tous les phénomènes sociaux, l'émigration est l'un des plus conformes à l'ordre de la nature, l'un des plus permanents à toutes les époques de l'histoire. Il est aussi naturel aux hommes --dit Burke-, d'affluer vers les contrées riches et propres à l'industrie, quand, pour une cause quelconque, la population y est faible, qu'il est naturel à l'air comprimé de se précipiter dans les couches d'air raréfié »l . Ce passage que nous empruntons au savant économiste $\mathrm{M}$. Paul Leroy-Beaulieu, et où se trouve de plus cité un nom d'une haute valeur politique, exprime une pensée qu' on ne discute plus aujourd'hui. Pour la plupart des pays d'Europe, l'émigration est non seulement un fait, mais un besoin annuel, c'est la saignée salutaire qui empêche la pléthore.

Le large courant d'émigrants qui part de l'Ancien Monde suit la marche naturelle de l'humanité ; il se dirige à l'ouest. Au delà de l'Atlantique, deux grands continents, connus de la grande majorité des hommes depuis quatre siècles seulement, offrent encore de larges espaces inoccupés, des richesses incalculables à peine en exploitation. Le courant, qui varie de forme et d'intensité avec les époques, a actuellement deux bras principaux: l'un se dirige à l'ouest et déverse son flot de population sur les États-Unis, l'autre, plus récent, oblique fortement au sud et pénètre dans l'estuaire du Rio de la Plata d'oú il inonde la République Argentine. Dans les premiers siècles qui suivirent la découverte de l'Amérique, le courant d'émigration était autre. Unique, il se dirigeait sur l'archipel des Antilles, et, de là, rayonnait sur les régions comprises entre les deux tropiques.

Le changement actuel de direction s'explique facilement. Ce que la grande masse des émigrants du $x^{2}{ }^{e}$ et du $x^{2}{ }^{e}$ siècle allaient chercher en Amérique, c'était la fortune facile, c'était la mine d'or qui leur permît de revenir riches au bout de quelques années en Europe, et de finir leurs jours dans leur pays natal. Ce que l'émigrant d'aujourd' hui

\footnotetext{
${ }^{1}$ De la colonisation chez les peuples modernes, par M. Paul Leroy-Beaulieu, page 467. Paris, Guillau$\min$ et $\mathrm{C}^{\mathrm{ie}}$.
} 
désire, c'est un coin de terre pour y bâtir sa maison, des champs pour les ensemencer, ce n'est pas la fortune inespérée, c'est une tranquille aisance, c'est l'oubli de la misère et de la lutte terrible pour l'existence ; c'est, en un mot, le calme et la paix au sein d'une nouvelle patrie. On comprend que, dans ces conditions, l'Européen se porte de préférence vers les régions tempérées au nord et au sud des tropiques qui lui rappellent davantage la patrie abandonnée et lui évitent de passer par une acclimatation quelquefois difficile. Ce fut naturel aussi à l'origine, puisque, réputées autrefois moins riches en métaux précieux, elles étaient moins peuplées que les régions tropicales et offraient plus de champ libre à la colonisation.

Toutefois le moment actuel est un moment critique. Les ÉtatsUnis et la République Argentine commencent -malgré leurs immenses territoires encore inoccupés - à se peupler outre mesure sur certains points. On oppose déjà des digues, dans le premier de ces deux pays, au flot de l'émigration. Contrarié dans sa marche, celui-ci est obligé de chercher une autre route que celle qu'il suivait depuis bien longtemps. Où se dirigera-t-il ? Nous croyons le moment venu pour lui de reprendre son cours primitif. Les pays de l'Amérique espagnole ont perdu leur réputation d'Eldorado ; ils gardent leur fertilité. Ils auront toujours la vraie richesse, celle du sol, sans cesse renaissante. D'immenses régions n'attendent que la pelle et la pioche pour produire non les trésors qu'on leur a demandés pendant longtemps, mais des récoltes abondantes ; non de l'or, mais des moissons dorées.

Entre toutes les républiques latines de l'Amérique, Costa Rica occupe certainement un des premiers rangs, si on les classe suivant les avantages et les ressources que chacune d'elles offre à l'émigration. Peu de pays ont en perspective un plus brillant avenir. Son climat est salubre, tempéré, et comparable à un perpétuel printemps ; la fertilité de son sol a fait sa richesse actuelle et la garantit ; sa position est certes privilégiée ; la faiblesse numérique de sa population lui permettra d'accueillir pendant longtemps les étrangers, non comme une charge, mais comme un bienfait. 
Où trouver, en effet, un meilleur terrain pour l'émigration et l'emploi fructueux des capitaux étrangers ? L'agriculture manque de bras, elle les demande à grands cris et plus elle en aura à sa disposition, plus elle sera la source féconde de la richesse non seulement de quelques-uns, mais du pays tout entier. Les terres en friche sont nombreuses, d'une fertilité reconnue, et leur prix en permet l' achat aux capitaux les plus minimes ; pour qui veut faire usage de ses bras, elles ne coûtent même que le travail de la mise en culture. La main d'œuvre de métier atteint un prix très élevé ; les bons ouvriers sont assurés d' une excellente rémunération de leur travail. L'activité industrielle a un large champ ouvert devant elle ; plusieurs industries s'introduiraient avec profit ; le développement de celles qui y sont déjà implantées donneraient de très beaux résultats. Les capitaux rendent au moins le double de ce qu'ils produisent en Europe, si l'on s'en rapporte uniquement au taux de l'argent ; ce rendement est beaucoup plus considérable si l' on considère le revenu des terres, le gain du commerce ou les bénéfices de l'industrie.

On parle beaucoup, il est vrai, en Europe de l'instabilité des gouvernements et du peu de sécurité qu'offrent les transactions dans les républiques hispano-américaines. Cette opinion est erronée en ce qui concerne Costa Rica. Le pays n'a presque pas connu les révolutions ; il est aujourd'hui sagement gouverné ; ses finances sont prospères et son état de civilisation le met à l'abri de tout changement rétrograde. On se représente aussi tous les pays de l'Amérique centrale, depuis l'isthme de Panama jusqu'au Mexique comme excessivement malsains. C'estune complète erreur : Costa Rica n'est pas Panama, ni la côte des Mosquitos. Le climat est au contraire, comme nous l'avons répété plusieurs fois, tout à fait salubre, plutôt tempéré que chaud. S'il existe encore dans le pays, comme partout, des régions malsaines, elles s'assainiront sans aucun doute, avec le défrichement, et, d'ailleurs les terres disponibles sont si nombreuses que, de longtemps encore, l'émigrant n'aura pas besoin de s'établir dans celles qui ne lui donneraient pas toute satisfaction. Une troisième erreur, trop répandue 
et que nous avons cherché à combattre en exposant l'état de civilisation auquel est arrivé Costa Rica, consiste à croire que ce pays est encore à moitié plongé dans la barbarie et l'ignorance. Cette appréciation est entièrement fausse ; l'aspect des villes, le caractère des habitants, les progrès de l'instruction publique, la sagesse administrative du gouvernement et le développement du commerce en sont la preuve. Le monopole de la civilisation n'appartient plus à l'Europe ; il est vrai que d'elle est sortie la lumière, mais le flambeau brille maintenant dans bien des endroits jadis plongés dans les ténèbres, et Costa Rica peut se vanter avec raison d'avoir atteint en peu d'années un degré de culture enviable.

On pourra faire à l'émigration pour Costa Rica deux objections plus sérieuses, mais qui ne sont point aussi redoutables qu'elles le paraissent à première vue. La première consiste en la cherté de la vie; la seconde naîtrait du souvenirdequelques tentatives malheureuses de colonisation risquées autrefois dans des conditions tout à fait déplorables.

La première objection tombe facilement. La vie est chère à Costa Rica, j'en conviens ; mais cette cherté est bien compensée par le taux des salaires. La vie est chère parce que le pays est riche ; il n'est pas besoin dans de longues considérations d'économie politique pour le prouver ; les États-Unis nous dispensent d'une démonstration inopportune. D'ailleurs tout n'est pas cher. L'Européen qui arrive dans le pays doit chercher à s'habituer promptement à la manière de vivre en usage. Ce qui est cher c'est de vouloir conserver intactes les coutumes de la vie européenne. L'émigrant d'Europe devra être supporter quelques privations qui ne dureront d'ailleurs qu' autant que l'habitude n'aura pas été perdue. Il fera bien, par exemple, de renoncer au vin qu'il peut boire en France ou à la bière qui ne lui coûte presque rien en Allemagne. Les haricots prendront la place des pommes de terre dans son alimentation principale. Mais sont-ce là de graves inconvénients? Nous ne le pensons pas, et nous n'aurions pas même parlé de règles de conduite aussi élémentaires, si l'expérience ne nous avait pas démontré plusieurs fois l'utilité d'insister sur ce point. 
Du fait, que quelques tentatives de colonisation à Costa Rica, n'ont pas donne les résultats qu'en attendaient ceux qui les avaient entreprises, on ne saurait déduire que l'émigration soit à déconseiller. La meilleure entreprise peut devenir malheureuse, si elle est mal conduite. Loin de nous, l'idée de jeter la pierre aux colons étrangers venus les premiers à Costa Rica. Ces tentatives datent d'un certain nombre d'années déjà et le pays n'était certes pas il y a, vingt ou trente ans, ce qu'il est aujourd'hui. Il leur fallut lutter donc contre des obstacles qui ont disparu maintenant.

Cependant nous croyons que si les projets avaient été plus longuement mûris, et le terrain mieux étudié, on n'aurait pas eu à regretter la non réussite des entreprises. C'est une grande erreur malheureusement trop commune- que de croire que les colonies se fondent d'un seul coup. A Costa Rica, pas plus qu'ailleurs, on ne saurait songer à voir villes et villages sortir de terre comme par enchantement, dans des lieux autrefois déserts. Certaines circonstances rares, l'affluence subite de la population dans une région riche en mines d'or, par exemple, permirent ces prodiges. Presque toujours les colonies ont d'humbles débuts. Les défrichements se font peu à peu. Il faut qu'un premier champ ait été ensemencé et produise déjà de quoi nourrir un certain nombre d'individus, pour qu'un groupe puisse se former et la colonie se constituer sans crainte de périr misérablement. Dans le cas exceptionnel cité plus haut, c'est au poids de l'or que les émigrants achètent de quoi subvenir à leurs premiers besoins. Mais les colons agricoles sont toujours pauvres à leur arrivée ; ils ne peuvent compter que sur le travail de leurs bras. En attendant que la forêt ait été jetée à bas, que les semences aient été confiées à la terre, que le premier grain de blé ou de maïs puisse être récolté, de quoi vivront-ils ? De quoi vivront-ils, surtout loin des habitations d'autres hommes, dans des régions encore à peu près désertes où les denrées alimentaires ne parviennent qu'avec la plus grande difficulté ? Ils souffriront mille maux ; ils perdront la confiance et l'espoir dont ils étaient armés à l'origine et, dégoûtés du travail, soupirant après la condition dans 
laquelle ils se trouvaient autrefois et dont ils ne voient plus que les beaux côtes, ils abandonneront l'un après l'autre leur dure entreprise. Ceciseul explique ce qui s'est passéà Costa Rica et ce qui s'y passerait encore si l'on y entreprenait l'e migration dans les mêmes conditions. Il ne faut pas songer à y transplanter des colonies entières tout d'un coup, à moins que ce ne soit dans un terrain préparé longtemps à l'avance, déjà en partie en cultivé et pouvant subvenir aux premiers besoins des nouveau venus.

Costa Rica cependant désire l'arrivée des émigrants. Nous avons même démontré plus haut qu'ils étaient pour ce pays presque une nécessité, et, en tout cas, une condition de sa prospérité future. En parlant des colonies, l'économiste que nous avons déjà cité dit : « Les seuls émigrants dont les colonies retirent quelque avantage, ce sont les jeunes gens vigoureux, pleins de courage et de patience ; les enquêtes anglaises ont prouvé qu'au-dessous de 16 ans et au dessus de 40 ans, l'émigration était plutôt une charge qu'une ressource pour les colonies " ${ }^{2}$. Quoiqu'il ne s' agisse pas ici de métropole et de colonies, nous nous rallions entièrement à l'opinion émise dans les lignes que nous venonsde transcrire. Ce qu'il faut à Costa Rica, ce sont des jeunes gens ou des hommes faits, tant qu'ils ne dépassent pas une certaine limite d'âge ; mais il les faut surtout vigoureux, pleins de courage et de patience. Une erreur assez commune chez les émigrants consiste à croire que la vie sera de toute manière plus facile pour eux dans les pays d'outre-mer. Nous n'hésitons pas à le dire, elle est souvent plus dure à l'origine. L'Amérique offre cet avantage que la sueur y est plus féconde, que le travail y est plus rémunérateur et qu'une fois les premières difficultés vaincues, l'aisance s'y acquiert plus facilement. Mais celle-ci ne s'obtient pas sans peine ; il faut de la vigueur, il faut du courage et de la persévérance.

Il faut autre chose encore. L' homme faible, le lâche on l'inconstant ne gagneront pas même leur vie ; celui qui ne sait rien faire ne réussira

P. Leroy-Beaulieu, ouv. cir., p. 481. 
pas davantage. Costa Rica est un pays neuf, mais c'est aussi un pays qui progresse chaque année. On exige de l'étranger non seulement qu'il travaille bien, mais encore qu'il travaille mieux que l'ouvrier indigène. S'il se montre bon artisan, bon cultivateur, bon industriel, son mérite sera promptement reconnu ; s'il travaille mal ou même médiocrement, on se détournera de lui non sans raison. Il faut aussi avoir plus d'une corde à son arc. Dans un pays où les bras manquent, on est souvent obligé de se mettre à tout. Chacun ne doit compter que sur son intelligence et sur ses forces.

Malgré tous les avantages qu'on pourrait lui off rir, l'émigrant ne doit pas non plus arriver sans ressources. Quelque soit le travail qu'il compte entreprendre, des fonds lui sont toujours nécessaires pour son premier établissement. Et pour attendre les premiers gains, s'il ne veut pas être à la charge d'autrui ou commencer par contracter une dette qu'il n'éteindra ensuite pas facilement. De toute manière, un petit capital lui assurera l'indépendance ; le manque complet de ressources propres risque fort de l'empêcher de l'acquérir de longtemps.

Une bonne immigration ne comptera donc dans ses rangs ni gens faibles, ni personnes inutiles, ni individus complètement misérables. Ceux-là ne réussiront pas à Costa Rica. Les forts, les persévérants, les habiles, ceux qui possèdent quelques ressources y ont, au contraire, leur avenir assuré. On désire surtout des agriculteurs, des artisans sachant bien leur métier et des ouvriers industriels capables d'entreprendre eux-mêmes les mille petites industries productives qui manquent encore dans le pays.

Les pays qui font appel à l'immigration ont toujours pris des mesures pour aider les émigrants. Costa Rica n'entend pas rester en arrière sur ce point. Comme preuve à 1 'appui, nous pouvons citer le fait suivant: à la fin de 1 'année 1888 , plus d'un millier d'Italiens occupés aux travaux de la ligne du chemin de fer en construction abandonnèrent leur travail demandant à être rapatriés ${ }^{3}$. Désireux de

\footnotetext{
${ }^{3}$ Nous n'avons pas à juger le différend survenu entre l'entreprise du chemin de fer et les Italiens qui avaient été engagés pour les travaux. Nous dirons cependant qu'aucun d'eux n'a eu le moindre sujet de
} 
garder, en partie du moins, dans le pays cette immigration déjà sur place, le gouvernement offrit aussitôt aux Italiens, qui le désireraient, de faire venir leurs familles à ses frais. Quelques-uns acceptèrent l'offre et sont aujourd'hui définitivement établis à Costa Rica. La grande majorité cependant préféra regagner ses foyers à la suite d'un arrangement conclu avec une agence maritime. La décision du gouvernement à l'égard des familles italiennes peut être prise de nouveau en faveur d'autres émigrants. Nous croyons de même pouvoir affirmer que promptement une somme assez importante du budget sera destinée à encourager l'immigration, soit en payant le passage des émigrants, soit en leur assurant à leur arrivée ici, des moyens de subsistance immédiats.

De toute manière les renseignements seront faciles à prendre. Costa Rica possède des consuls dans les principales villes d'Europe et des Etats-Unis. Ils fourniront à ceux qui en feront la demande toutes les explications désirables et transmettront volontiers à leur gouvernement les propositions qui leur seront faites par les personnes qui désirent s'expatrier. Monsieur le Consul général résidant à Paris se chargera spécialement de recevoir les demandes et c'est à lui qu'on devra s'adresser de préférence ${ }^{4}$.

Nous nous tenons nous-mêmes à la disposition de tous ceux qui voudraient des détails plus précis, sur des points qui pourraient les intéresser particulièrement. Nous serons très heureux, si ce que nous avons exposé, sans réticence ni exagération d'aucun genre, est utile aux hommes qui cherchent une nouvelle patrie.

\footnotetext{
plainte vis à vis la population de Costa Rica qui les a secourus et hébergés pendant plusieurs semaines avec un dévouement digne d'éloges. Quelques journaux d'Europe ont accueilli des correspondances qui faisaient retomber sur le pays tout entier les infortunes des ouvriers, triste conséquence de l'abandon des travaux. Comme témoin oculaire des évènements, nous croyons de notre devoir de démentir formellement ces récits mensongers qui ne prouvent que la noire ingratitude de leurs auteurs.

${ }^{4}$ Ecrire à M. E. Palacios, Consul Général de Costa Rica en France, rue des Petites Ecuries, 28, Paris.
} 